\title{
Miranda
}

Revue pluridisciplinaire du monde anglophone /

Multidisciplinary peer-reviewed journal on the English-

speaking world

$20 \mid 2020$

Staging American Nights

\section{Les nuits américaines de Mihail Malaimare}

\section{Jocelyn Dupont}

\section{QpenEdition}

\section{Journals}

Édition électronique

URL : http://journals.openedition.org/miranda/25366

DOI : 10.4000/miranda.25366

ISSN : 2108-6559

\section{Éditeur}

Université Toulouse - Jean Jaurès

\section{Référence électronique}

Jocelyn Dupont, « Les nuits américaines de Mihail Malaimare », Miranda [En ligne], 20 | 2020, mis en ligne le 24 mars 2020, consulté le 16 février 2021. URL : http://journals.openedition.org/miranda/ 25366 ; DOI : https://doi.org/10.4000/miranda.25366

Ce document a été généré automatiquement le 16 février 2021.

\section{c) (i)}

Miranda is licensed under a Creative Commons Attribution-NonCommercial-NoDerivatives 4.0 International License. 


\title{
Les nuits américaines de Mihail Malaimare
}

\author{
Jocelyn Dupont
}

\section{Introduction}

1 La question de la mise en scène de "la nuit américaine" dans un contexte cinématographique invite d'emblée à comprendre cette locution à travers ses résonances polysémiques; il s'agit de l'entendre, d'une part, comme l'exploration dramatique du nocturne et des thématiques qu'on lui rattache spontanément (brouillage, irrationnel, énigme, intime, secret, obscur) et, d'autre part, de considérer la nuit américaine comme effet dramaturgique liée au dispositif du cinéma, comme le rappelle d'ailleurs spontanément le titre du célèbre film de François Truffaut (1973), hommage au cinéma dans son ensemble et à l'art de la lumière en particulier. La nuit américaine est trompeuse, et son obscurité fallacieuse avant tout un effet de lumière :

La lumière qu'on observe à l'œil va impressionner la pellicule négative devant la caméra, qui elle-même va être transposée sur de la pellicule positive. Ensuite seulement, la lumière du projecteur va nous restituer l'image sur l'écran. La lumière que nous aurons observée à l'œil nu sur les acteurs va subir un certain nombre de traitements et d'interprétations, avant d'être restituée devant les spectateurs. Les agents de transposition sont nombreux. (Loiseleux 59)

Qui, au cinéma, fait la lumière, et comment faire lumière sur la lumière ? Et comment, par quelque lumineux paradoxe, faire nuit ? C'est-à-dire, selon la plus simple acception de la locution, plonger dans le noir, dans l'obscurité, dans l'absence de lumière. Impossible, bien entendu. Ainsi, "une image de la nuit ne sera jamais qu'une vision interprétée par une lumière qui la représente, la suggère ou la sublime » (38).

Day for night, la nuit américaine, « relève des effets spéciaux, c'est l'effet qui consiste à tourner de jour une scène extérieure de telle façon que le spectateur ressente, à la projection, l'impression d'une scène de nuit » (Passek 550). Trope cinématographique, la nuit américaine relève donc de la rhétorique de l'obscurité - l'asaphéia de Démétrios en ce qu'elle est «une obscurité obtenue, dans un but purement artistique, par une 
manipulation avisée du langage » (Montandon 1214), en l'occurrence cinématographique.

4 Et qui donc parle le langage de la lumière ? L'auteur? Le cinéaste ? Le réalisateur ? Rappelons avec Jacques Loiseleux que «la lumière est un médium d'expression à la disposition du réalisateur pour exprimer une idée avec le cinématographe » (51) et que c'est au directeur de la photographie - cinematographer en anglais, c'est-à-dire très littéralement celui qui écrit le mouvement - qu'il revient d'éclairer ou, dans le cas qui nous intéresse, d'assombrir le film. Une certaine tendance de la critique de cinéma, depuis plus d'un demi-siècle et l'avènement de la politique des auteurs, a relégué, du moins aux yeux du grand public, les directeurs de la photographie ou «chefs opérateurs » dans l'ombre de l'auteur, instance originaire au moins aussi fantasmatique que l'image manquante de notre « nuit sexuelle», pour reprendre la formule de Pascal Quignard. Ce fantasme originaire n'est pas nouveau et n'appartient pas au seul domaine du cinéma - il a été plutôt importé de la littérature avant d'y être transposé. Quoi qu'il en soit, l'éclipse est plus souvent totale que partielle.

Dans les pages qui suivent, il sera question de trois films récents de Francis Ford Coppola, " auteur » par excellence au sens où aime à l'entendre une certaine tradition critique française, notamment portée par Les Cahiers du Cinéma depuis le milieu des années 1950. Selon ce modèle théorique largement établi quoique sujet à débat ${ }^{1}$, l'auteur transcende les contraintes du système hollywoodien, parvenant ainsi à « imprimer" sa personnalité sur un genre et une production (Bordwell et Thomson, 777-8). Ainsi, «le film est considéré comme une expression directe de sa personnalité [et] l'auteur devient plus important que le film lui-même : la signature certifie la valeur de l'œuvre, elle en garantit la qualité.»(Casetti 90). Si la célèbre "politique des auteurs " a dans un premier temps porté aux nues le travail de cinéastes américains tels Samuel Fuller, Nicholas Ray ou installés au cœur du système hollywoodien tels Otto Preminger et, bien entendu, Alfred Hitchcock, ce modèle d'analyse fécond a connu un regain d'intensité au milieu des années 1970 avec l'émergence des cinéastes du « Nouvel Hollywood» tels Steven Spielberg, Brian de Palma, Martin Scorsese, sans oublier Francis Ford Coppola, envers lequel le rédacteur-en-chef actuel Stéphane Delorme n'a jamais caché son admiration². Au-delà de ce seul effet de signature, les trois longs-métrages dont il va être ici question ont tous en commun d'avoir été " mis en lumière" par le même directeur de la photographie, Mihail Malaimare, chef opérateur roumain inconnu du grand public et âgé d'une trentaine d'années à peine lors de sa première collaboration avec Coppola en 2007. On l'aura compris, c'est au moins autant de la poétique des nuits américaines du Roumain que des rêveries de l'Italo-américain qu'il sera question. Pour autant, l'objet de cet article n'est pas de chercher à déstabiliser ni à véritablement critiquer un paradigme théorique qui a fait ses preuves de longue date. L'auteur, ainsi que le souligne Jean-Claude Biette, par sa position moderne de responsabilité, maintient sa place dans le processus de production cinématographique et l'«emprise » relative qu'il exerce sur son texte filmique lui permet de revendiquer «l'urgence de formuler une vérité ou un ensemble de vérités personnelles»(16). L'efficacité poétique du modèle ne sera donc pas franchement remise en cause. Nonobstant, un film ne se "signe" pas seul, et au fil du développement qui suit ces propos introductifs, notre intention principale est de braquer le projecteur aussi sur celui qui a, très matériellement, su faire la lumière des nuits américaines dans les trois films réalisés par Francis Ford Coppola depuis 2007. 


\section{Coppola en habit de lumière}

6 Rappelons d'emblée que Coppola n'a jamais manqué d'audace quand il s'est agi d'illuminer ses films. Certaines de ses collaborations avec des chefs opérateurs restent mémorables, à l'instar de celle qui donna naissance à One From the Heart [Coup de Coeur] (1981), romance entièrement éclairée aux néons, dont la photographie à l'esthétique très eighties fut assurée par Ronald V. Garcia, directeur de la photographie pour Twin Peaks : Fire Walk with Me de David Lynch (1991). On peut également citer le noir et blanc expressionniste ponctué d'irruptions de couleurs dans Rumble Fish [Rusty James] (1984) signé Steven Burhum, chef opérateur qui collabora longtemps par la suite avec Brian de Palma, et enfin la flamboyance empourprée de Dracula (1997) dont la photographie fut signée par Michael Ballhaus (1935-2017), directeur de la photographie attitré de Martin Scoscese et à qui l'on doit notamment l'esthétique 'néo-noir' de Goodfellas [Les Affranchis] et le très rougeoyant Gangs of New York (2003). Coppola, cinéaste protéiforme pour qui le besoin de renouvellement du langage cinématographique est constant, n'a jamais hésité à s'emparer de la lumière et de la couleur pour donner à l'image un surcroît d'artificialité, promouvant ainsi un cinéma baroque auquel il a souvent été associé. Il sait surtout laisser une très grande marge de manœuvre à ses directeurs de la photographie, qui souvent lui en ont su gré, à l'instar de Mihail Malaimare qui reconnaissait dans un entretien accordé aux Cahiers du Cinéma en 2009 que : «Coppola donne beaucoup de liberté à trouver le langage visuel qui convient » (Malaimare 2009, 21).

$7 \quad$ Les trois films en question sont Youth Without Youth [L'homme sans âge], sorti en salles en 2007 après un hiatus de presque dix ans - le film précédent, The Rainmaker [L'idéaliste] était par ailleurs une commande - suivi de près par Tetro (2009) et enfin Twixt, sorti sur les écrans au milieu de l'année 2011. Quoique très différents en termes diégétiques et esthétiques, ces trois films participent néanmoins du même geste créateur et sont tous les trois principalement filmés en mode nocturne. Si la lumière, comme nous l'avons déjà écrit, est bien un "médium d'expression à la disposition du réalisateur pour exprimer une idée » en collaboration avec son chef opérateur, force est de constater que l'expressivité trouvée par Coppola et Malaimare repose avant tout sur un défi jeté à la lumière, tant l'obscurité et la nuit y sont prégnantes, « délibérément interprétée(s), théâtralisée(s) (...) dans le souci d'une esthétique favorable à la narration » (Loiseleux 40).

8 Youth Without Youth, Tetro et Twixt constituent une trilogie singulière composée à partir d'une méthode identique qui fait de chacun des films un exemple assez caractéristique de ce qu'on peut qualifier de film "d'auteur", selon ce biais exégétique conduisant à voir le metteur en scène comme instance démiurgique ayant tout contrôle sur son objet filmique. Malgré le caractère illusoire de ce modèle d'analyse, il n'est pas faux de constater que pour chacun des films de la trilogie, on semble toucher à des cas-limites d'autorité filmique eu égard au cahier des charges auto-imposé régissant chacune des réalisations.

9 Premièrement, au-delà de simples "thèmes » et motifs que l'on peut toujours, fut-ce par excès rhétorique, ramener à la soi-disant «sensibilité » de l'auteur, chaque film doit être lié à une expérience singulière de Coppola en personne: lecture, autobiographie, ou rêve - nous y reviendrons. Deuxièmement, le scénario doit être 
signé par Coppola lui-même, y compris dans le cas d'une adaptation de texte littéraire. Troisièmement, et c'est sans doute ici le point crucial, le film doit être produit de manière autonome, sans aucun apport financier d'un quelconque producteur ou studio tiers. Pour les trois films concernés, c'est American Zoetrope, le propre studio de Coppola, fondé en 1969, qui fut l'unique financeur, garantissant ainsi à ce dernier un pouvoir décisionnaire total sur le produit filmique final.

Aussi démiurgique un réalisateur soit-il, il ne saurait néanmoins prétendre à être " l'auteur", au sens étymologique du terme, de chacun des aspects de son film. Rares sont en effets les cinéastes qui peuvent prétendre à la maitrise de chacune des quatre étapes principales de la genèse d'un film - écriture, réalisation, montage, postproduction. Même Stanley Kubrick, parangon de l'auteur total du cinéma américain, savait s'entourer d'une équipe de collaborateurs fidèles, dont le directeur de la photographie John Alcott, l'homme de la lumière kubrickienne qui passa pourtant, du moins aux yeux du grand public, l'essentiel de sa carrière dans l'ombre du cinéaste tout-puissant. Il en va de même pour Coppola, dont les collaborateurs - à l'exception peut-être de son monteur Walter Murch, quoique la carrière de ce dernier fût si riche et si diverse qu'il semble quelque peu réducteur de le qualifier comme tel - sur l'ensemble d'une carrière presque longue de six décennies, furent toutefois plus divers que ceux de Kubrick, à commencer par ses directeurs de la photographie.

11 Pour ce qui est de Mihail Malaimare, on peut même parler d'une collaboration débutée sous le signe de la rencontre heureuse. Malaimare fut en effet retenu par Coppola à l'issue d'un étrange casting de chefs opérateurs organisé à Bucarest en 2007 au cours de la préparation du tournage de Youth Without Youth, le premier des trois volets de la trilogie dont il demeura par la suite le seul et unique directeur de la photographie. Youth Without Youth situe son intrigue principalement dans la capitale roumaine.

12 Au cours de l'année 2005, pendant la phase profilmique et les essais de casting, le réalisateur et son équipe réduite s'étaient installés à Bucarest, plus ou moins incognito, dans un local mis à la disposition du réalisateur. Coppola avait donc fait le choix de s'y installer avec son équipe, pour "chercher en Europe centrale, antipodes et lieu originaire de Hollywood, la possibilité de devenir celui qui, sachant tout, ayant tout fait, s'inventerait lui-même en débutant» (Frodon 11). Ce qualificatif, s'il ne sied à Coppola que dans la mesure où l'on a à faire avec lui à un cinéaste soucieux de renouveler son art à chacun de ses films, était en revanche particulièrement approprié pour ce qui concernait alors les états de services de Malaimare. À l'époque, ce dernier n'avait travaillé que sur quatre films, dont deux courts-métrages, exclusivement en Roumanie. De manière assez peu conventionnelle, les auditions d'acteurs s'accompagnaient d'" auditions » de directeurs de la photographique, lesquels étaient chargés à chaque fois d'éclairer la scène d'essai ${ }^{3}$. À l'issue d'une de ces séances de test, Malaimare, dont le jeune âge ne semble pas avoir été le moindre des atouts pour éclairer un film principalement construit sur l'idée d'un retour vers une jeunesse impossible, finit par l'emporter aux yeux du réalisateur. Pour autant, et bien qu'ils partagent néanmoins une prédilection pour le mode nocturne et les effets de nuit américaine, ces trois films possèdent des esthétiques bien distinctes. Il n'est donc pas faux d'affirmer que la trilogie personnelle de Francis Ford Coppola devient aussi celle de Mihail Malaimare, au gré de leurs expérimentations dans l'art de cette «représentation transposée » de la nuit qu'est la nuit américaine (Loiseleux 42). 


\section{Youth without Youth : le bleu de la nuit du langage}

13 Youth without Youth est l'adaptation d'un court roman de 1976 écrit par Mircea Eliade (1907-1986), figure de proue de l'histoire des religions, dont les études sur la mythologie comparée, les travaux sur le sacré et le profane (1959) ainsi que sur la théorie de l'éternel retour (1949) ont durablement marqué leur époque, même si aujourd'hui elles peuvent apparaître un peu datées et trop imprégnés d'ésotérisme. La dimension fictionnelle de l'œuvre d'Eliade est en revanche plus confidentielle; c'est d'ailleurs plus ou moins par l'entremise d'une connaissance intime - Wendy Doniger, professeure d'études religieuses comparées à l'université de Chicago, ancienne disciple d'Eliade et amie d'enfance de Coppola - que cette novella finit par atterrir entre les mains du cinéaste et de le convaincre qu'il s'agit là de le transformer en projet filmique. Le film, dont l'action se déroule à Bucarest au seuil de la Seconde Guerre mondiale, se situe aux frontières du fantastique pour constituer un exemple assez rare de ce qu'on pourrait qualifier de "linguistique-fiction" plus encore que de fiction linguistique 4 . Il narre les égarements et les paradoxes temporels de son protagoniste Dominic Matei (interprété par Tim Roth), linguiste de renom, obsédé par les origines du langage et, après avoir été frappé par la foudre dans la séquence d'ouverture, perdu - de même que trop souvent le spectateur - dans une intrigue à multiples rebondissements, oscillant entre «récit faustien et thriller politique " (Niogret 49). Néanmoins, le cœur du récit est riche d'enjeux philosophiques et symboliques, conformément à ce que l'on pouvait attendre d'une fiction écrite par Eliade. Il s'agit en effet, ni plus, ni moins, de la quête insensée d'un proto-langage, de «cette autre foudre qui fit passer nos ancêtres du grognement au logos» (Tessé 17), convoitée, dans l'espace de la diégèse, par les belligérants soucieux de s'approprier ce secret si bien gardé des dieux. Le film, tourné en grande partie avec une caméra numérique HD (la SonyF900), possède une esthétique singulière, parfois quelque peu surannée, d'autres fois plus audacieuse, comme si Coppola n'avait pas su tout à fait trancher entre le choix de la nouveauté et les capacités de la lumière numérique d'une part, et les hommages à un certain expressionnisme européen de l'autre. L'intention poétique demeure bien réelle néanmoins, et comme a pu l'observer Hubert Niogret :

Francis Ford Coppola n'est pas un cinéaste réaliste, et, dans ses meilleures réalisations, il a voulu chercher à transcender la réalité de la matière cinématographique qu'il avait entre les mains pour atteindre une poésie cinématographique. Plus que certains de ses collègues réalisateurs américains qui laissent la porte ouverte à l'imaginaire dans leur dramaturgie, c'est dans la matière même du cinéma, ses outils, les éléments physiques à sa disposition pour construire un langage que Coppola trouve son expression, celle de ses rêves. (49)

La matière, et les éléments physiques permettant d'atteindre à cet imaginaire en question, fût-il rêvé (et ce sera à nouveau le cas avec Twixt), Youth without Youth semble en partie les avoir trouvés dans les séquences de nuit américaine. Le film les multiplie : on en compte au moins cinq et elles sont suffisamment longues et importantes en termes diégétiques pour diffuser leur aura bleuté sur l'ensemble de sa durée. Plutôt conventionnelles, elles n'ont pas été tournées en numérique mais en pellicule $35 \mathrm{~mm}$ (à partir de Tetro, le $35 \mathrm{~mm}$ sera totalement abandonné au profit du numérique HD). Celles-ci accordent une prévalence à la couleur bleue, caractéristique des nuits américaines depuis que celles-ci ont rencontré le cinéma en couleurs dans l'âge classique hollywoodien. Toutefois, les séquences en nuit américaine - day for night - de 
Youth without Youth privilégient un bleu assez outré. Ni les filtres utilisés, ni l'étalonnage ne cherchent à atténuer le phénomène optique de Purkinje selon lequel, en très faibles éclairements, l'œil est surtout sensible au bleu (Passek 551) alors que les lumières de la nuit et de la lune tirent naturellement vers le jaune. Au contraire, l'image est volontairement saturée de bleu. Il en résulte une succession de séquences durant lesquelles le spectateur se retrouve happé dans une lumière bleutée et irréelle, si bien que la dimension onirique l'emporte sur la simple mise en scène de la nuit. Il s'agit en outre souvent de scènes intimes, plus propices aux chuchotements qu'aux cris, bien que ce soit proche de la jouissance orgasmique que, lors d'une de ces séquencesclé, le personnage de Laura (Alexandra Maria Lara), retrouve le son primal, protolinguistique, celui-là même qui inscrit l'homme dans l'ordre du symbolique, lui donne ainsi conscience du temps - et de la mort. C'est donc dans le bleu profond des nuits américaines de Bucarest que Coppola trouve l'expression filmique de la quête fondamentale entreprise avec son vingtième long-métrage. Conte philosophique, linguistique et chromatique souvent complexe voire méandreux, Youth without Youth inaugure ainsi de manière assez inattendue - car en apparence si éloignée de l'intime, contrairement aux deux volets qui suivront - la trilogie personnelle de Coppola.

\section{Tetro : ou comment faire lumière sur la nuit antérieure}

« En este momento, mi luz es la verdad » (Tetro, 45’18)

Tetro, au titre programmatique - l'adjectif signifie 'sombre' en espagnol - est un film à la lumière étrange, entre « hommage au Hollywood des années 50 et libertés formelles presque expressionnistes» (Delorme 21). Le film a été tourné en numérique HD avec une caméra différente de cette utilisée pour Youth without Youth. Une fois encore, il s'agissait d'une expérience nouvelle pour Malaimare, qui sut s'adapter à la révolution technologique alors en cours à la fin des années 2000 et qui allait voir la quasiintégralité des réalisateurs et des "écrivains du cinéma", à commencer par les directeurs de la photographie, abandonner définitivement la pellicule pour le tout numérique. Tetro est principalement tourné en noir et blanc, le plus souvent en scènes de nuit ou en nuit américaine, que ce soit en day for night ou même en night for night. Ce choix est d'autant plus surprenant que le film situe l'essentiel de son action dans le quartier de La Boca à Buenos Aires, connu notamment pour ses façades bariolées et ses couleurs vives et chaudes. Dans un entretien qu'il a accordé aux Cahiers du cinéma, Malaimare revient d'ailleurs sur le chromatisme presque excessif de l'environnement fourni par la capitale argentine, déclarant que «les couleurs sont si puissantes en Argentine qu'on finit par ne plus distinguer clairement les effets de texture » (Malaimare 2009, 22).

En noir et blanc et principalement composé de scènes de nuit, Tetro s'ingénie donc à déjouer les propriétés chromatiques d'un décor naturel. L'effet recherché est d'ailleurs rapidement obtenu, puisque le spectateur oublie vite tout ce que Buenos Aires pourrait offrir de "pittoresque " pour se concentrer sur le cœur de l'intrigue, à savoir la quête identitaire entreprise par Bennie (Alden Ehrenreich), venu retrouver son frère aîné Tetro (Vincent Gallo) et les secrets d'une paternité impossible à assumer. L'intrigue du film est bien trop sinueuse pour qu'on puisse se risquer ici à tenter de la résumer, mais disons qu'il s'agit surtout, pour ce benjamin en quête de père, de faire lumière sur la zone d'ombre qui entoure une certaine période de son existence et surtout sur la 
disparition inexpliquée de son frère aîné pour un exil imposé. Au milieu de l'opacité ambiante, la lumière et ses papillonnements sont d'emblée donnés comme la métaphore structurelle du film. Coppola déclare ainsi :

La métaphore la plus importante est liée à la lumière. C'est le premier plan: le personnage qui regarde l'ampoule électrique, avec le papillon. La lumière symbolise la vérité et la bonté, mais elle devient aussi un symbole de mort à cause des phares de la voiture qui aveuglent Tetro après l'accident de sa mère. Elle vous attire comme un papillon, et peut vous tuer. C'est la lumière des projecteurs, de la célébrité, du succès. (Coppola 17)

On est aussi tenté d'ajouter que c'est aussi la lumière d'une vérité trop brûlante qui invite à rester tapi au cœur de la nuit argentine, comme le fait le très hermétique personnage de Tetro durant la première moitié du film, opaque à toute tentative d'" éclaircissement " que son frère tente d'apporter à son sujet. Car le récit familial est hanté par les fantômes transgénérationnels aussi bien que par Éros et Tanathos.

«La nuit infernale est obscure (éternelle). Mort. /La nuit utérine est invisible (temporelle). Naissance.", écrit Pascal Quignard dans La nuit sexuelle (82). Et Tetro d'égrener ses douloureuses vérités, fondées précisément sur trois morts et une naissance, et sur une impossible étreinte transgressive - la " nuit antérieure ", l'image manquante, le traumatisme et l'impossible «figuration originaire ${ }^{5}$ » que les tentatives d'écriture théâtrale autobiographique de Bennie mèneront vers une insoutenable vérité. À ces phosphorescences douloureuses s'oppose donc l'ombre, comme le refoulé, opacifié, s'oppose au retour intrusif de la mémoire. C'est la raison pour laquelle de nombreuses séquences du film sont très peu éclairées, a fortiori lorsqu'il s'agit de scènes de nuit. Techniquement, le numérique HD permet des images de très bonne qualité même en faible éclairage - ce qui n'était pas encore le cas lors du tournage de Youth without Youth trois ans auparavant. Le dispositif demeurait au final très réduit. Il n'en était pas moins d'une grande efficacité.

19 Tetro est un film qui place le rapport au point aveugle du souvenir au cœur de sa diégèse et de ses images, alliant mode nocturne et hétérogénéité iconique. Le travail sur la nature de l'image cinématographique paraît essentiel en ce qu'il permet de souligner presque physiquement l'hétérogénéité du matériau traumatique dans l'économie générale du récit. Le souvenir traumatique - notamment depuis Abraham et Torok - a souvent été comparé à un fantôme, un intrus, un shrapnel mental que le corps ne peut ni introjecter, ni expulser, mais dont il n'est pas possible de se défaire. Tetro s'empare de cette altérité et, manipulant les facettes de ce "diamant noir ${ }^{6}$ ", en fait l'un de ses enjeux cinématographiques fondamentaux.

20 L'aspect le plus évident du rapport de ce film à la matérialité de l'image est évidemment le choix du noir et blanc pour le récit filmique principal tandis que les flashbacks ou les séquences analeptiques sont en couleurs. Cette alternance n'a en soi rien de très original, et Coppola avait d'ailleurs déjà exploré la coexistence du noir et blanc et de la couleur dans Rumble Fish. Mais le travail du réalisateur et de son équipe ne s'est pas arrêté à cette simple distinction. Avec l'aide de Malaimare, le cinéaste s'est penché sur la matérialité même de l'image-souvenir et sur la façon de l'inscrire dans le récit principal, mimant ainsi l'enjeu même de la cure du trauma, à savoir l'inscription du matériau traumatique hétérogène dans une chaîne symbolique cohérente. C'est bien à un processus d'inscription de l'hétérogène que nous assistons lorsque nous voyons les images-souvenirs en couleurs s'intégrer peu à peu au récit en noir et blanc (Dupont). Et c'est également une forte expérience de disruption perceptuelle qui se manifeste au 
spectateur lorsqu'apparaissent à l'écran, dans un format et une texture différents, les images ressurgies du passé.

21 Lors des essais de préproduction, plusieurs procédés furent envisagés par Coppola et son chef opérateur afin d'intégrer le matériau mémoriel dans la texture du film tout en soulignant son hétérogénéité: les lentilles Lomo, produites en URSS dans les années 1980, dont le rendu chromatique est particulièrement vif et patiné ou encore l'utilisation d'une pellicule Anscochrome, assez proche du Technicolor, avec des effets de saturation des couleurs, un procédé utilisé notamment dans les films de famille des années 50 et 60 aux États-Unis.

Pour faire écho à la citation d'Hubert Niogret concernant les expérimentations entreprises dans Youth without Youth, notons que des tentatives de superposition matérielle de plusieurs images furent également effectuées, en utilisant des images de pellicules $16 \mathrm{~mm}$ incrustées dans le récit filmique en numérique. Une dernière remarque enfin. Nous avons déjà indiqué que le film a été tourné en support numérique HD, avec seulement deux caméras. Toutefois, pour la scène fatidique de l'accident dans laquelle Tetro perd sa mère, pas moins de cinq caméras furent utilisées. Parmi elles, une $35 \mathrm{~mm}$ qui ne fut sortie que pour cette scène, et dont les images, bien qu'elles existent, ne furent finalement pas exploitées dans le montage final, comme s'il avait paru essentiel, pour figurer « la nuit sexuelle » fondée par le traumatisme de l'accident telle qu'elle s'écrit dans Tetro, d'évacuer la matrice de toutes les images cinématographiques classiques, à savoir la pellicule $35 \mathrm{~mm}$.

La mythologie grecque nous apprend que la Déesse Nix, parée de son grand manteau dont elle recouvre le monde, eut deux fils: Hypnos et Thanatos (Quignard 82). Ce dernier, nous l'avons vu, gouverne Tetro, nous avons pu le constater. Obscur jusqu'à son signifiant-titre, le film n'a de cesse qu'il renvoie à l'opacité invisible de la nuit utérine (Eros) finalement infernale (Thanatos). Éros le gouverne, mais Thanatos veille aussi sur le point d'origine d'une naissance marquée par, deux fois, du sceau de la mort de la mère : la mère biologique de Tetro, et celle de Bennie.

\section{Twixt : comment rêver la nuit américaine}

Twixt, troisième volet de la trilogie, sorti un peu plus de deux ans après Tetro est, dans sa genèse même, placé sous le signe du rêve - donc sous l'égide d'Hypnos. L'origine du film est à la fois onirique et psychopompique. L'anecdote qui la décrit, et que l'on retrouve non seulement dans la bouche de Coppola lui-même mais aussi dans celle de ses collaborateurs, mérite d'être rappelée. Après avoir bouclé Tetro, Coppola était parti en repérage à Istanbul, ville chère à son cœur dans laquelle il comptait réaliser son troisième volet de sa trilogie. À l'issue d'une soirée passablement arrosée au raki (l'alcool anisé local), il s'effondre dans sa chambre d'hôtel. Cette nuit-là, il fait un rêve étrange - un rêve, confiera-t-il ensuite aux Cahiers du cinéma lors de la promotion de Twixt, « tellement précis et original qu'il pourrait donner naissance à un film » :

Je me trouvais au milieu d'une forêt, terrifié, et c'est alors que cette jeune fille est sortie de la forêt, une jeune fille très étrange avec de grandes dents tordues couvertes de bagues. Dans le rêve, elle me provoquait: "Est-ce que je te fais peur?»-, comme si elle était un vampire. Et puis, je suis arrivé dans ce vieil hôtel avec une tombe, d'où sont sortis plein d'enfants pour jouer, en pleine nuit, comme si c'était l'après-midi. Pendant que je fais cette expérience, Edgar Allan Poe fait son 
apparition, et il veut m'apporter son aide. Tout s'est passé dans le rêve exactement comme dans le film. (Coppola 11) filmer un rêve? Notons en outre que les séquences oniriques reposent sur ce que l'on pourrait appeler le paradoxe du rêve nocturne, remarquant avec Bernard Dieterle que «le rêve, phénomène nocturne, a en effet rarement un contenu nocturne, on rêve durant la nuit mais rarement de la nuit » (Montandon 1199). Gageure, donc, que de transposer à l'écran non seulement "l'impression de la nuit " mais aussi l'impression d'un rêve. À ce premier paradoxe il convient d'en ajouter un second, propre à la réalité technique de la poïétique de la nuit américaine: en effet, aussi intense le degré d'obscurité recherché soit-il, il nécessitera forcément un excédent de lumière : « «le paradoxe de la nuit américaine, c'est qu'on a besoin du jour pour créer la nuit.» (Malaimare 2012, 26).

choix lumineux et chromatiques insolites effectués par Coppola et Malaimare plongent à plusieurs reprises le spectateur dans des atmosphères nocturnes où s'allient esthétique gothique, surréalisme et fantasmagorie. Comme l'a déclaré Malaimare :

Les séquences [de rêve] se déroulent la nuit, dans la forêt. Après un premier planning, on a constaté que l'on devrait passer les trois quarts du temps en travail nocturne. Alors, environ deux semaines avant le début du tournage, Francis m'a dit qu'il aimerait essayer des nuits américaines un peu spéciales - pas celles, dans les règles, que l'on avait dans Youth without Youth. Restait à trouver quoi. (25)

Comme Tetro, Twixt a été entièrement tourné en images numériques, ce qui a permis un certain nombre d'effets, notamment grâce à l'utilisation de calques, une fonctionnalité assez élémentaire du logiciel Photoshop. Coppola et Malaimare ont ainsi longuement travaillé à un chromatisme incongru voire impossible, n'ayant de cesse d'" aberrer " l'image (Loiseleux 44). Véritable gant jeté à la figure de l'effet de Purkinje, les nombreuses séquences de nuit américaine privilégient le rouge et le jaune, couleurs normalement impossibles à obtenir en tournage de nuit américaine sur pellicule classique.

Autant d'incongruités d'éclairage qui caractérisent Twixt et en font à ce jour un film où la mise en scène de la nuit touche au paroxysme de l'originalité et de l'onirisme. Il est par ailleurs intéressant de noter qu'alors que le réalisateur et le directeur de la photographie sont exactement les mêmes que ceux qui ont travaillé aux nuits américaines de Youth without Youth, jamais les deux films ne pourraient être plus dissimilaires dans leurs effets de mise en scène de la nuit. Pour ce qui est du dernier opus, les détails techniques, à jamais hors d'atteinte pour les profanes - dont l'auteur de ces pages se réclame, bien malgré lui - mêlent effets spéciaux en production et en postproduction, maquillages fluorescents et pailletés ${ }^{7}$, rotoscopie et étalonnage contre nature. Ensemble, ils finissent d'altérer les nuits américaines de Twixt, dont les scènes nocturnes, proposent une incursion toute particulière dans le fantastique. Nuit et rêve $\mathrm{y}$ « collaborent pour déréaliser le réel, [devenant] de fait les alliés objectifs de la folie » (Montandon 1200), à l'instar de celle d'un Edgar Poe qui, comme Malaimare, n'ignorait pas les arcanes de la rhétorique de l'obscurité. 


\section{Conclusion}

En à peine plus de cinq ans, soit un laps de temps remarquablement concentré, Francis Ford Coppola a donc offert au public une "trilogie d'auteur " tout à fait singulière par ses enjeux personnels et esthétiques, attestant ainsi l'incroyable vitalité d'une œuvre qui, depuis plus d'un demi-siècle, explore sans relâche et avec gusto les potentialités du langage cinématographique. Pourtant cette quête, ainsi que nous pensons l'avoir expliqué, creuse un sillon souvent plus secret qu'ouvertement spectaculaire. La trilogie aux résonances si profondément intimes n'aurait toutefois pu être portée vers la lumière sans le travail du jeune chef opérateur Mihail Malaimare, dont cet article espère avoir pu démontrer combien les "nuits américaines" relèvent de l'écriture cinématographique et de ses potentialités poétiques davantage que de la simple mise en scène d'un "effet spécial ». Pourtant, nous avons pu observer que la dimension technique de la nuit américaine demeurait un aspect essentiel de la composition cinématographique, a fortiori au cours des années 2010, période cruciale pour le cinéma qui a basculé vers le "tout numérique ", de la pré - à la postproduction. C'est sans doute ici que la conscience aiguë de la nature technologique de son art - explorée sans relâche depuis The Conversation (1974) - a permis à Coppola de conserver une actualité et une modernité esthétique jusqu'à ce jour, en s'entourant notamment de collaborateurs précieux et renouvelés, à l'instar de Malaimare. Si l'on peut gager que ce brillant directeur de la photographie ne pourra jamais véritablement aspirer au statut de «co-auteur » des trois films dont il vient d'être question, tant le modèle de l'auteur cinématographique demeure fondé sur la promotion d'une singularité individuelle comme "unique responsable » du produit filmique et comme "témoignage d'une personnalité, [...] lieu où se déposent sentiments, obsessions et pensées d'un individu qui s'exprime avec les images et les sons » (Casetti 91), il n'en demeure pas moins que la présente analyse a permis de montrer comment l'écriture de la nuit américaine, dont la poétique est si déterminante sur les trois films ici à l'étude, était loin d'être assignable, précisément, à quelque "unique responsable». Du bleu profond et secret de Youth without Youth à l'opacité brillante de Tetro, jusqu'aux aberrations chromatiques des rêveries poesques de Twixt, Coppola et Malaimare ont su composer ensemble un remarquable triptyque nocturne, véritable ode à la nuit américaine, dont les potentialités demeurent aussi intensément obscures que lumineuses.

\section{BIBLIOGRAPHIE}

Films

Youth Without Youth, dir. Francis Ford Coppola, Zoetrope, 2007.

Tetro, dir. Francis Ford Coppola, Zoetrope, 2009.

Twixt, dir. Francis Ford Coppola, Zoetrope, 2011.

Ouvrages cités et consultés

Miranda, 20 | 2020 
Biette, Jean-Claude. Qu'est-ce qu'un cinéaste? Paris : P.O.L, 2000

Bordwell, David et Christine Thompson. L'art du film 3e édition française. Traduction Cyril Béghin, Bruxelles : De Boecq, 2014.

Casetti, Francesco. Les théories du cinéma depuis 1945. Traduit de l'italien par Sophie Saffi. Paris : Armand Colin, 2012.

Coppola, Francis Ford. « Entretien avec Francis Ford Coppola ». Cahiers du cinéma 651 (décembre 2009) : 15-20.

Dupont, Jocelyn. « Incandescence du traumatisme dans Tetro de Francis Ford Copolla ». Sillages critiques [En ligne], 19 | 2015. URL : <http://sillagescritiques.revues.org/4341>

Dutel, Jérôme. « Linguistique-fiction et fictions linguistiques ». Thèse de doctorat inédite, Université Jean Moulin Lyon 3, 2007.

Frodon, Jean-Michel. « La troisième rose entre les dents ». Cahiers du cinéma 627 (novembre 2007) : 11.

Loiseleux, Jacques. La Lumière en cinéma. Paris : Cahiers du Cinéma, 2004.

Malaimare, Mihail. «L'Argentine et l'argentique. Entretien avec Mihail Mailaimare ». Cahiers du cinéma 651 (décembre 2009) : 21-24.

---. « Des Ultraviolets d'enfer. Entretien avec Mihail Mailaimare ». Cahiers du cinéma 677 (avril 2012) : 24-28.

Montandon, Alain (ed.) Dictionnaire littéraire de la nuit. Paris : Honoré Champion, 2013.

Niogret, Hubert. « L'homme sans âge. Modernité et nostalgie ». Positif 561 (novembre 2007) : 48-9.

Passek, Jean-Loup (ed.) Dictionnaire du cinéma. Paris : Larousse, 1998.

Quignard, Pascal. La nuit sexuelle. Paris : Flammarion, 2007.

Tessé, Jean-Philippe. « Coup de foudre ». Cahiers du cinéma 627 (novembre 2007) : 13.

\section{NOTES}

1. On pourra, entre autres, consulter avec profit les études approfondies que Jean-Claude Biette a consacrées à la question dans ses deux recueils de textes : Poétique des auteurs (Cahiers du Cinéma 1989) et Qu'est-ce qu'un cinéaste? (P.O.L. 2000).

2. Le succès de ce modèle d'analyse se poursuit de nos jours : comment, en effet, considérer Gus Van Sant, Jim Jarmusch, Quentin Tarantino, David Lynch ou Terrence Malick autrement que comme des auteurs? La question de "l'autorité " dans l'art cinématographique demeure fascinante mais dépasse toutefois largement le propos de cet article.

3. Dossier de presse de Youth Without Youth

4. Dans sa thèse intitulée «Linguistique-fiction et fictions linguistiques ", Jérôme Dutel explique que pour un récit soit considéré comme relevant de la linguistique-fiction, «les langues ou les langages imaginaires et les extrapolations imaginaires qu'il proposent, imposent ou présupposent, doivent former le nœud dramatique, le moteur de l'intrigue, de l'histoire et de l'œuvre, pour cristalliser des virtualités linguistiques. [...] Il est possible de dire qu'en linguistique-fiction la théorie linguistique n'est pas l'élément central de l'intrigue, c'est l'intrigue elle-même » (132-3). Une telle définition convient parfaitement à l'intrigue de Youth Without Youth. 
5. Sans doute l'expression si souvent utilisée de nos jours de « scène primitive » est-elle excessive pour désigner le fait que les hommes et les femmes dérivent d'une étreinte entre un homme et une femme autres qu'eux-mêmes puisque cette étreinte est nécessairement invisible à leur regard faute d'être déjà conçus. Peut-être faut-il lui préférer l'expression de "nuit sexuelle » puisqu'à n'en pas douter il est plus question dans l'âme inquiète d'une nuit antérieure que d'une figuration originaire. (Quignard 101, c'est moi qui souligne)

6. Nous reprenons cette locution expressive et significative à Stéphane Delorme qui en fit l'intitulé de sa critique de Tetro dans le numéro 651 des Cahiers du cinéma (décembre 2009).

7. Inspirés notamment des recherches effectuées par Henri-Georges Clouzot dans ses essais pour son film inachevé L'Enfer.

\section{RÉSUMÉS}

Cet article s'intéresse aux modalités de la nuit américaine, entendue comme effet spécial de la dramaturgie cinématographique d'une part, et d'exploration d'une certaine poétique de l'obscur de l'autre, dans trois longs métrages récents du cinéaste Francis Ford Coppola. On y montre comment l'association d'une vision auctoriale et d'une technicité permettent, au sein d'une seule et unique trilogie, de parcourir une gamme d'effets chromatiques et poétiques. Cet article propose aussi une réflexion sur le rôle de la lumière, ou de son absence, dans l'art cinématographique

This article deals with the different modalities of night scenes in three recent movies by Francis Ford Coppola. Whether shot in day for night or night for night, the author explores the poetics of darkness and reverie set forth in each of the movies in question. The aim of this article is to show how filmic authority cannot depart from cinematography, and a close alliance between director and cinematographer. It also offers some elements of reflection concerning the role of light, or its absence, in film art.

\section{INDEX}

Keywords : day for night, darkness, Francis Ford Coppola, Mihail Malaimare, Youth Without Youth, Tetro, Twixt, author theory (in film), cinematography

Mots-clés : nuit américaine, obscur, Francis Ford Coppola, Mihail Malaimare, Youth Without Youth, Tetro, Twixt, auteur (cinématographique), direction de la photographie

\section{AUTEURS}

\section{JOCELYN DUPONT}

Maître de conférences

Université de Perpignan, CRESEM Axe Textes (EA

7379)

Jocelyn.dupont@univ-perp.fr 